\title{
A Theory of Inertia Based on Mach's Principle
}

\author{
Volkmar Putz ${ }^{+}$ \\ Institute for General Secondary Education, University College of Teacher Education Vienna (PH Wien), \\ 1100 Vienna, Austria; volkmar.putz@phwien.ac.at \\ + Current address: Grenzackerstrasse 18, A-1100 Vienna, Austria.
}

Received: 8 July 2019; Accepted: 12 August 2019; Published: 16 August 2019

Abstract: A non-relativistic theory of inertia based on Mach's principle is presented as has been envisaged, but not achieved, by Ernst Mach in 1872. The central feature is a space-dependent, anisotropic, symmetric inert mass tensor. The contribution of a mass element $d m$ to the inertia of a particle $m_{0}$ experiencing an acceleration from rest is proportional to $\cos ^{2} \alpha$, where $\alpha$ is the angle between the line connecting $m_{0}$ and $d m$ and the direction of the acceleration. Apsidal precession for planets circling around a central star is not a consequence of this theory, thereby avoiding the prediction of an apsidal precession with the wrong sign as is done by Mach-like theories with isotropic inert mass.

Keywords: inertia; Mach's principle; history of physics

PACS: $04.20 . \mathrm{Cv}$

\section{Introduction}

In 1872 Ernst Mach formulated his famous principle about the inert mass of a particle, which he assumed to be induced somehow by the presence of other masses in the universe [1]. Particularly, Mach criticized Newton's concept of absolute space as demonstrated by the famous bucket gedankenexperiment which concludes that true rotational motion cannot be defined as the relative rotation of the body with respect to the immediately surrounding bodies. In fact, if a bucket filled with water starts to rotate around its vertical axis, the surface of the water will at first (when the water has not yet started to move) stay flat. After a while, the surface of the water will assume a concave shape as it acquires the motion of the rotating bucket. For Newton this shows that true rotational motion cannot be defined as the relative rotation of a body (here the water) with respect to the immediately surrounding bodies (here the bucket): When there is a relative movement between water and bucket (at the beginning of the experiment), no centrifugal forces act on the water; however, when the relative movement has disappeared (when the water moves synchronously with the bucket) centrifugal forces are there.

However, the argument neglects (gravitational) influence from bodies farther away. In fact, Mach argues that only relative motion is established: "Newton's experiment with the rotating vessel of water simply informs us that the relative rotation of the water with respect to the sides of the vessel produces no noticeable centrifugal forces, but that such forces are produced by its relative rotations with respect to the mass of the earth and other celestial bodies." (Ernst Mach, as quoted by [2]).

There are many possible formulations of Mach's principle (especially in the context of general relativity, [3]), some being considered more strongly Machian than others. Bondi and Samuel have listed eleven statements that can be called Mach principles [4]. For our considerations, the following five formulations are the most relevant:

- The universe, as represented by the average motion of distant galaxies, does not appear to rotate relative to local inertial frames. 
- An isolated body in otherwise empty space has no inertia.

- Local inertial frames are affected by the cosmic motion and distribution of matter.

- Inertial mass is affected by the global distribution of matter.

- Overall rigid rotations and translations of a system [universe] are unobservable.

A rather weak formulation of Mach's principle is that the motion of matter in one place should affect which frames are inertial in another. Thereby, the inertia of a mass is a phenomenon induced solely by the gravitational influence of other masses. In a universe without gravity there would be also no inertia. Unfortunately, Mach never formulated his ideas as a physical theory.

In this paper, I want to present a theory consistent with Mach's principle. The theory is non-relativistic. Hence it is more of a historical value, giving what Mach did not (or could not) deliver nearly 150 years ago, when Mach's ideas were not yet overgrown by Einstein's theories (which, contrary to Einstein's hopes and struggles, do not fully incorporate the Mach principle, [4]).

A central aspect of the theory is the anisotropy of the inert mass, which therefore has to become a tensor. In the 1950s, experiments in search of anisotropy of inertia have been performed with negative result [5]. Later, these experiments have been recognized as not suitable, ignoring the fact that locally not only the test particle but all masses (including those of the experimental setup) exhibit the same anisotropy of inertia [6,7]. Different experiments where this effect does not matter are still to be performed.

\section{Results}

\subsection{Assumptions}

We start with the following assumptions, which are all very simple and plausible:

- Assumption 1: A single point mass in an otherwise empty universe has no defined movement, i.e., no inertia, no acceleration, no momentum or kinetic energy.

- Assumption 2: Two-point masses in an otherwise empty universe can have a defined movement only along their connecting line. Any movement at a constant distance between the masses cannot be defined. This comprises rotation about each other (no tangential inertia) and collective translation. No kinetic energy or momentum arises due to such movement: Inertia, acceleration, momentum or kinetic energy can only be defined with respect to increasing/reducing the distance between the masses, i.e., for radial movement. We would like to have a theory where the sign of this radial movement does not matter.

- Assumption 3: Since the inertia of a test particle is 'created' by the gravitational forces of other masses, it will depend on the direction. Therefore the angle between the direction of the test particle movement and the connecting line to any inducing mass will be crucial.

- Assumption 4: The size of the induced inertia should decrease with increasing distance $r$ between test particle and inducing mass as $1 / r^{a}$ with $0<a<2$. Here $0<a$ guarantees vanishing inertia at infinite distance (which, of course, is an assumption itself). $a \geq 2$ would lead to large effects perceivable in daily life, since anisotropy of inertia would balance or even excel the effects of Newton's gravity. In fact, $a=1$ is a standard assumption in literature, see e.g., [8], where, however, isotropy of inertia is postulated.

- Assumption 5: The inertia of a test particle within a hollow sphere of constant mass density is isotropic and constant. If the mass distribution is largely isotropic within our universe, this assumption guarantees that this theory is very similar to our daily experience. 


\subsection{Theory}

A definition of the kinetic energy of a test particle with gravitational mass $m_{0}$ within a universe with other (gravitational, inertia inducing) masses $m^{\alpha}$, which is consistent with all assumptions above is given by (summation convention is used for the spatial indices $i, j$ ):

$$
\begin{gathered}
E_{k i n}=\frac{1}{2} \sum_{\alpha} v_{i}^{\alpha} M_{i j}^{\alpha} v_{j}^{\alpha}, \\
M_{i j}^{\alpha}=M_{j i}^{\alpha}=\gamma m_{0} m^{\alpha} \frac{r_{i}^{\alpha} r_{j}^{\alpha}}{\left|\vec{r}^{\alpha}\right|^{3}},
\end{gathered}
$$

where $\gamma$ is a natural constant, presumably connected with Newton's gravitational constant, $\vec{r}^{\alpha}=$ $\vec{x}_{0}-\vec{x}^{\alpha}$ is the distance between $m_{0}$ and $m^{\alpha}$, with $\vec{x}_{0}$ and $\vec{x}^{\alpha}$ the absolute positions of $m_{0}$ and $m^{\alpha}$ in space, respectively. Similarly, $\vec{v}^{\alpha}=\dot{\vec{x}}_{0}-\dot{\vec{x}}^{\alpha}$ is the relative (not only radial!) velocity between $m_{0}$ and $m^{\alpha}$ (as will be the relative acceleration $\dot{\vec{v}}^{\alpha}=\vec{a}^{\alpha}$ later on). The sum is over all masses $m^{\alpha}$ in the universe with the exception of $m_{0}$.

The corresponding components of the momentum and force of the test particle $m_{0}$ are then given by

$$
\begin{gathered}
p_{i}=\frac{\partial E_{k i n}}{\partial\left(\vec{v}_{0}\right)_{i}}=\sum_{\alpha} M_{i j}^{\alpha} v_{j}^{\alpha}, \\
F_{i}=\frac{d p_{i}}{d t}=\sum_{\alpha}\left(\dot{M}_{i j}^{\alpha} \sigma_{j}^{\alpha}+M_{i j}^{\alpha} a_{j}^{\alpha}\right) .
\end{gathered}
$$

If and only if we are in a coordinate system where all inducing masses $m^{\alpha}$ are at rest, we have $\vec{v}^{\alpha}=\vec{v}_{0}$ and the formulae simplify to

$$
E_{k i n}=\frac{1}{2} \vec{v}_{0}^{T} \overleftrightarrow{M} \vec{v}_{0}, \quad \vec{p}=\overleftrightarrow{M} \vec{v}_{0}, \quad \overleftrightarrow{M}=\gamma m_{0} \sum_{\alpha} m^{\alpha} \frac{\vec{r}^{\alpha}\left(\vec{r}^{\alpha}\right)^{T}}{\left|\vec{r}^{\alpha}\right|^{3}}
$$

\subsection{Check of Assumptions}

Let us now investigate how this definitions fulfil Assumptions 1-5. For this, we do not consider Newtonian gravitation beyond the induction of inertia.

Assumption 1. Since there exists only $m_{0}$ and not one single $m^{\alpha}$, the $M_{i j}^{\alpha}$ are all zero. There is no kinetic energy and no momentum and also no force.

Assumption 2. For translations at a constant distance, $\vec{v}^{\alpha}=0$. For (possibly non-uniform) rotations of a mass $m_{0}$ with constant radius $R$ around a single point mass $m^{\alpha}$ (or vice versa), we assume $\vec{x}^{\alpha}=(0,0,0) \Rightarrow$ $\vec{r}^{\alpha}=\vec{x}_{0}-\overrightarrow{0}=(R \cos \omega t, R \sin \omega t, 0)$, with $\omega$ possibly time dependent. Thus we have

$$
\begin{gathered}
\overleftrightarrow{M}=\frac{\gamma m_{0} m^{\alpha}}{R}\left(\begin{array}{ccc}
\cos ^{2} \omega t & \sin \omega t \cos \omega t & 0 \\
\sin \omega t \cos \omega t & \sin ^{2} \omega t & 0 \\
0 & 0 & 0
\end{array}\right) \\
\vec{v}^{\alpha}=R(\dot{\omega} t+\omega)\left(\begin{array}{c}
-\sin \omega t \\
\cos \omega t \\
0
\end{array}\right) .
\end{gathered}
$$

With our definitions above one sees easily that $\vec{p}=\overrightarrow{0}, E=0$ and again there is no force (this is also true if $m^{\alpha}$ and $m_{0}$ rotate synchronously around some other rotational center, e.g., both being fixed onto one single watch hand). Especially, there are no centripetal or centrifugal forces. (Therefore, 
the gravitational attraction between the two masses will eventually lead to a collision, regardless if there is a rotation or not.)

Assumption 3. Anisotropy of inertia is realized in our definition. As an illustration, let us consider a simple experiment involving three masses, see Figure $1 . m_{0}$ is at rest in $(0,0,0)$. We have two masses $m^{\alpha}$, with mass $m^{1}=M$ at $(0,-R, 0)$ and mass $m^{2}=2 M$ at $(-R, 0,0)$.

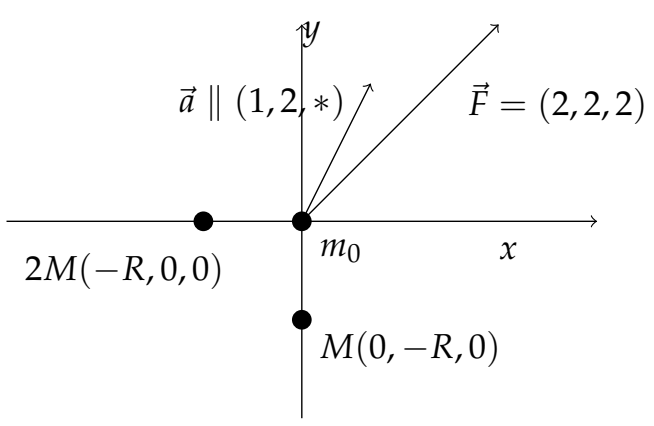

Figure 1. Simple example for the check of Assumption 3.

After a short calculation, we find (with $\vec{p}_{0}=0$ )

$$
\overleftrightarrow{M}=\frac{\gamma m_{0} M}{R}\left(\begin{array}{lll}
2 & 0 & 0 \\
0 & 1 & 0 \\
0 & 0 & 0
\end{array}\right), \quad \vec{F}=\overleftrightarrow{M} \vec{a}
$$

If a force $\vec{F}=(2,2,2)$ is applied, this will lead to an acceleration of $\vec{a}=(1,2, *)$, which is clearly not the same direction as $\vec{F}$. The * signals the not defined acceleration in the here inertia-free $z$-direction.

If $\vec{p}_{0} \neq \overrightarrow{0}$ at the beginning, things become more complicated, since the change of $\vec{r}^{1}$ and $\vec{r}^{2}$ (the position of $m_{0}$ relative to the two other masses) gives extra terms leading to $\vec{a} \nVdash(1,2, *)$.

Assumption 4. One sees easily that the contribution of each mass $m^{\alpha}$ to $\overleftrightarrow{M}$ is proportional (besides the dependence on direction) to $1 /\left|\vec{r}^{\alpha}\right|$.

All these considerations can easily be generalized to systems with more masses $m^{\alpha}$, where one also has e.g., the absence of kinetic energy and momentum if all masses move uniformly or the absence of centrifugal forces if all masses rotate uniformely without change of relative distances. This corresponds perfectly to Mach's famous bucket-gedankenexperiment.

Assumption 5. It is perhaps the most crucial and definitely the most complicated to prove the following. In Appendix A, we will give the detailed calculation (A2). The result for the inert mass tensor of a test particle $m_{0}$ (resting or moving with constant velocity relative to the sphere) within a thin sphere of radius $R$ and constant area mass density $\sigma$ (and hence $4 \pi R^{2} \sigma$ its overall mass) is:

$$
\overleftrightarrow{M}=\frac{\gamma}{3} \frac{4 \pi R^{2} \sigma}{R} m_{0}\left(\begin{array}{lll}
1 & 0 & 0 \\
0 & 1 & 0 \\
0 & 0 & 1
\end{array}\right)
$$

This corresponds to a constant, isotropic inertia independent of the spatial position (as long as within the sphere) and would, therefore, be the physical setting for the emergence of usual Newtonian inertia. The calculation in Appendix A shows that the contribution of a mass element $d m$ to the inertia of a particle $m_{0}$ at rest (experiencing an acceleration) is proportional to $\cos ^{2} \alpha$, where $\alpha$ is the angle between the line connecting $m_{0}$ and $d m$ and the direction of the acceleration. So if the angle is $90^{\circ}$, 
there is no contribution of $d m$ to the inertia, whereas the contribution is maximal if both directions are in parallel. Thus, apsidal precession for planets circling around a central star is not a consequence of this theory (leaving room for additional effects due to general relativity), but it avoids the prediction of an apsidal precession with wrong sign as is done by Mach-like theories with isotropic inert mass (proportional e.g., to the local gravitational potential), [8,9].

\section{Conclusions}

In this paper, a non-relativistic theory of inertia based on Mach's principle was presented. The theory is by no means intended to replace Einstein's general theory of relativity, e.g., it will not explain perihelion precession of planets since the gravitation of the central star (at this scale nearly a point mass) has barely any influence on the tangential inertia of surrounding objects. However, this theory may come to life at the scale of galaxies and their movement. In any case, the next step will be to (try to) incorporate this theory into general relativity.

The most crucial task would be a proper testing of inert (an)isotropy using experiments which do not rely only on masses (i.e., gravitation and inertia but also e.g., electric fields. Since induced inertia and Newtonian gravitation always come together, interference of both effects may complicate things.

Funding: This research received no external funding.

Conflicts of Interest: The author declares no conflict of interest.

\section{Appendix A. Calculation of M within a Sphere}

We start with a test particle at the origin of our coordinate system. This particle is within a thin sphere of constant area mass density $\sigma$ with center $(0|0|-a)$ and radius $R(R>a)$, see Figure A1. We use a coordinate system where this sphere is at rest (which does not mean that the sphere is physically 'at rest' as this is meaningless in an otherwise empty universe, see Assumption 1).

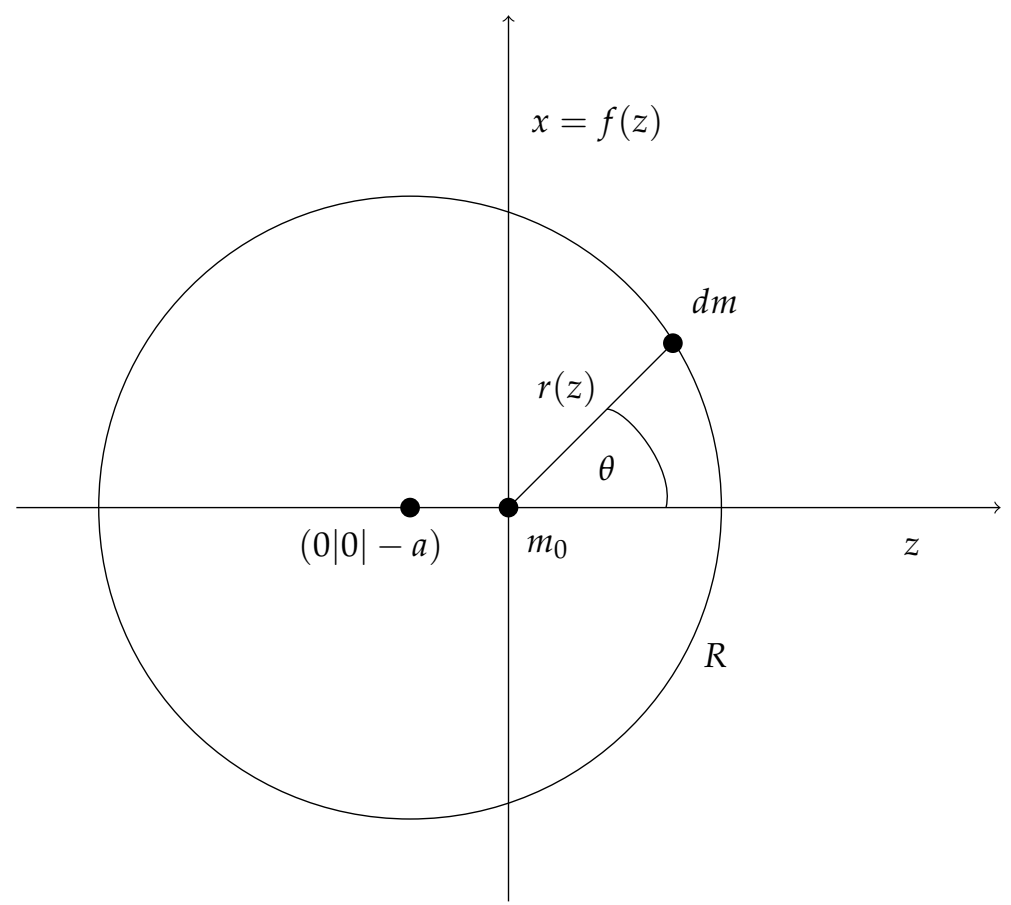

Figure A1. Simple example for the check of Assumption 5. 
The mass element $d m$ of the sphere is at point $(z, x=f(z), 0)$, where

$$
\begin{aligned}
f(z) & =\sqrt{R^{2}-(z+a)^{2}}, \\
f^{\prime}(z) & =-\frac{z+a}{\sqrt{R^{2}-(z+a)^{2}}}, \\
f(z) \sqrt{1+f^{\prime 2}(z)} & =\sqrt{R^{2}-(z+a)^{2}} \sqrt{1+\frac{(z+a)^{2}}{R^{2}-(z+a)^{2}}}=R, \\
r(z) & =\sqrt{z^{2}+f^{2}(z)}=\sqrt{R^{2}-a^{2}-2 a z} .
\end{aligned}
$$

For later use, we want to calculate the gravitational potential $\Phi_{\text {in }}$ within the sphere:

$$
\begin{aligned}
m_{0} \Phi_{i n} & =\int_{0}^{2 \pi} d \phi \int_{-R-a}^{R-a} d z \frac{m_{0} \gamma \sigma}{r(z)} \underbrace{f(z) \sqrt{1+f^{\prime 2}(z)}}_{R} \\
& =2 \pi R m_{0} \gamma \sigma \int_{-R-a}^{R-a} \frac{d z}{\sqrt{R^{2}-a^{2}-2 a z}} \\
& =\left.2 \pi R m_{0} \gamma \sigma \frac{-1}{a} \sqrt{R^{2}-a^{2}-2 a z}\right|_{-R-a} ^{R-a} \\
& =\frac{2 \pi R m_{0} \gamma \sigma}{a}(\underbrace{\sqrt{R^{2}-a^{2}+2 a R+2 a^{2}}}_{R+a}-\underbrace{\sqrt{R^{2}-a^{2}-2 a R+2 a^{2}}}_{R-a}) \\
& =4 \pi R m_{0} \gamma \sigma .
\end{aligned}
$$

Of course, this result is not at all surprising: The gravitational potential within a hollow sphere of homogeneous mass density is constant, i.e., independent of the position.

We now want to calculate $\overleftrightarrow{M}$ at the coordinate origin (where $m_{0}$ is located):

$$
\begin{aligned}
& \overleftrightarrow{M}=\int_{\text {sphere }} d^{3} r m_{0} \gamma \sigma \frac{\vec{r} \vec{r}^{T}}{|\vec{r}|^{3}} \\
& =\int_{0}^{2 \pi} d \phi \int_{-R-a}^{R-a} d z \frac{m_{0} \gamma \sigma}{r^{3}(z)} \underbrace{f(z) \sqrt{1+f^{\prime 2}(z)}}_{R}\left(\begin{array}{ccc}
x^{2} & x y & x z \\
y x & y^{2} & y z \\
z x & z y & z^{2}
\end{array}\right) .
\end{aligned}
$$

With $(x, y, z)=(r(z) \sin \theta \cos \phi, r(z) \sin \theta \sin \phi, r(z) \cos \theta)$ we see immediately that after the $\phi$-integration the off-diagonal terms are all zero and the diagonal terms yield $2 \pi$ for the $z^{2}$-term and $2 \pi / 2$ for the other two:

$$
\overleftrightarrow{M}=2 \pi R m_{0} \gamma \sigma \int_{-R-a}^{R-a} d z \frac{1}{r(z)}\left(\begin{array}{ccc}
\frac{1}{2} \sin ^{2} \theta & 0 & 0 \\
0 & \frac{1}{2} \sin ^{2} \theta & 0 \\
0 & 0 & \cos ^{2} \theta
\end{array}\right) .
$$

If the movement of the test particle is in the $z$-direction $\left(v_{0}=\left(0,0, v_{z}\right)\right)$ then (5) reduces to $E_{k i n}=\frac{1}{2} M_{z z} v_{z}^{2}$. One sees that the contribution of a mass element $d m$ to the inertia is proportional to $\cos ^{2} \theta$, where $\theta$ is the angle between the line connecting the test particle with $d m$ and the direction of test particle's movement. (Note that $d m=\sigma \cdot R \sin \theta d \phi \cdot \frac{d z}{\sin \theta}=\sigma R d \phi d z$ )

We start with the $z z$-term proportional to $\cos ^{2} \theta$. We rewrite:

$$
\cos ^{2} \theta=\left(\frac{z}{r(z)}\right)^{2}=\frac{z^{2}}{R^{2}-a^{2}-2 a z}
$$


and get:

$$
M_{z z}=2 \pi R m_{0} \gamma \sigma I_{z}=2 \pi R m_{0} \gamma \sigma \int_{-R-a}^{R-a} d z \frac{z^{2}}{\left(R^{2}-a^{2}-2 a z\right)^{3 / 2}},
$$

where $I_{z}$ means the integral without the prefactors. Here we use the well known formula [10]

$$
\begin{aligned}
& \int d z \frac{z^{2}}{(A z+B)^{3 / 2}} \\
& =\frac{2}{A^{3}}\left(\frac{1}{3}(A z+B)^{3 / 2}-2 B(A z+B)^{1 / 2}-B^{2}(A z+B)^{-1 / 2}\right),
\end{aligned}
$$

where in our case $A=-2 a$ and $B=R^{2}-a^{2}$ (note that the denominator is $r^{3}(z)>0$ ):

$$
\begin{aligned}
I_{z}=\int_{-R-a}^{R-a} \frac{z^{2}}{\left(R^{2}-a^{2}-2 a z\right)^{3 / 2}=} & -\frac{1}{4 a^{3}}\left(\frac{1}{3}\left(R^{2}-a^{2}-2 a z\right)^{3 / 2}\right. \\
& -2\left(R^{2}-a^{2}\right)\left(R^{2}-a^{2}-2 a z\right)^{1 / 2} \\
& \left.-\left(R^{2}-a^{2}\right)^{2}\left(R^{2}-a^{2}-2 a z\right)^{-1 / 2}\right)\left.\right|_{-R-a} ^{R-a} .
\end{aligned}
$$

We notice again

$$
\begin{aligned}
& \left(R^{2}-a^{2}-2 a(R-a)\right)^{1 / 2}=R-a, \\
& \left(R^{2}-a^{2}+2 a(R+a)\right)^{1 / 2}=R+a,
\end{aligned}
$$

and find:

$$
\begin{aligned}
I_{z}= & -\frac{1}{4 a^{3}}\left(\frac{1}{3}(R-a)^{3}-2\left(R^{2}-a^{2}\right)(R-a)-\frac{\left(R^{2}-a^{2}\right)^{2}}{R-a}\right) \\
& +\frac{1}{4 a^{3}}\left(\frac{1}{3}(R+a)^{3}-2\left(R^{2}-a^{2}\right)(R+a)-\frac{\left(R^{2}-a^{2}\right)^{2}}{R+a}\right) \\
= & \frac{1}{4 a^{3}} \cdot \frac{8}{3} a^{3}=\frac{2}{3}, \\
M_{z z}= & 2 \pi R m_{0} \gamma \sigma I_{z}=\frac{1}{3} \cdot 4 \pi R m_{0} \gamma \sigma .
\end{aligned}
$$

This is exactly one third of what we had before (A1), independent of the test particle's position within the sphere.

For the $x x$ - and $y y$-component, the integration is easy using $\frac{1}{2} \sin ^{2} \theta=\frac{1}{2}\left(1-\cos ^{2} \theta\right)$ with the results from above:

$$
2 \pi R m_{0} \gamma \sigma I_{x, y}=\frac{1}{2}(1-1 / 3) \cdot 4 \pi R m_{0} \gamma \sigma=\frac{1}{3} \cdot 4 \pi R m_{0} \gamma \sigma=I_{z} .
$$

Again, it is exactly $1 / 3$ of what we had before. In sum, we end up with

$$
\overleftrightarrow{M}=\frac{\gamma}{3} \frac{4 \pi R^{2} \sigma}{R} m_{0}\left(\begin{array}{lll}
1 & 0 & 0 \\
0 & 1 & 0 \\
0 & 0 & 1
\end{array}\right)
$$




\section{References}

1. Mach, E. Die Geschichte und die Wurzel des Satzes von der Erhaltung der Arbeit; Calve: Prague, Czech Republic, 1872.

2. Bouquiaux, L. Leibniz Against the Unreasonable Newtonian Physics. In Leibniz: What Kind of Rationalist; Dascal, M., Ed.; Springer: Berlin/Heidelberg, Germany, 2008; p. 104.

3. Barbour, J.; Pfister, H. Mach's Principle. From Newton's Bucket to Quantum Gravity; Birkhäuser: Basel, Switzerland, 1995.

4. Bondi, H.; Samuel, J. The Lense-Thirring effect and Mach's principle. Phys. Lett. A 1997, 228, 121-126. [CrossRef]

5. Huges, V.W. Gravitation and Relativity; Chiu, H., Hoffmann, W., Eds.; W. A. Benjamin, Inc.: New York, NY, USA, 1964; Chapter 6; pp. 106-120.

6. Anderson, J. Principles of Relativity Physics; Academic Press: Cambridge, MA, USA, 1967.

7. Dicke, R.H. Experimental tests of Mach's principle. Phys. Rev. Lett. 1961, 7, 359-360. [CrossRef]

8. Treder, H.-J. Die Relativität der Trägheit; Akademie: Berlin, Germany, 1972.

9. Treder, H.-J.; Mücket, J.-P. Große Kosmische Systeme; Akademie: Berlin, Germany, 1981.

10. Bartsch, H.-J. Taschenbuch mathematischer Formeln. Fachbuchverlag Leipzig 1997, 633.

(c) 2019 by the author. Licensee MDPI, Basel, Switzerland. This article is an open access article distributed under the terms and conditions of the Creative Commons Attribution (CC BY) license (http:/ / creativecommons.org/licenses/by/4.0/). 